\title{
Physical activity and social/cultural engagement as risk-reducing factors in the prevention of chronic pain in older age: findings from a longitudinal cohort study
}

\section{Dr Daisy Fancourt PhD \& Professor Andrew Steptoe DPhil DSc}

Department of Behavioural Science and Health, University College London, UK

Corresponding author: Dr Daisy Fancourt, 1-19 Torrington Place, London WC1E 7HB, d.fancourt@ucl.ac.uk

Background: Chronic pain is recognised as a major public health challenge as people age, and is linked with impaired physical capacity, falls, fatigue and depression. Yet, despite growing research on chronic pain management, there is little research into chronic pain prevention. Research suggests that combinations of physical and psychosocial factors could be protective against the development of chronic pain in older age. But there is still a clear need to identify specific multimodal activities that could be encouraged as part of a broader healthy lifestyle.

Methods: Using data from the English Longitudinal Study of Ageing we tracked adults aged 50+ who were free from chronic pain at baseline across a decade and explore whether physical activity (weekly moderate or vigorous activity) or psychosocial engagement (monthly visits to theatre/concert/museums or participation in community groups) reduced the risk of developing chronic pain. To confirm that pre-existing health conditions did not affect activity levels and predispose individuals to develop chronic pain, we further excluded those individuals with a chronic health condition at baseline (e.g. arthritis/cancer/COPD/diabetes/stroke/angina), providing an analytic sample size of 2,062 (51.2\% female, mean age 62.4 years). We used logistic regression analyses adjusted for identified socio-economic, health and social confounders and weighted for differential non-response.

Findings: Over the 10 years, $41.6 \%(n=966)$ of participants experienced moderate-severe chronic pain and for $21.9 \%(n=508)$ this lasted for more than 2 years. Engaging in vigorous weekly activity was protective against the development of chronic pain $(\mathrm{OR}=0.76, \mathrm{SE}=0.08,95 \% \mathrm{Cl}=0.62$ to 0.93 ) when controlling for all identified confounders. But no effects were found for moderate weekly activity. Monthly cultural engagement was also protective against the development of chronic pain $(O R=0.77$, $\mathrm{SE}=0.08,95 \% \mathrm{Cl}=0.62$ to 0.95$)$, but community group participation was not. Sensitivity analyses found no evidence of reverse causality.

Interpretation: This study supports previous work suggesting that vigorous (but not moderate) physical activity can be protective against the development of chronic pain in older age, and showed for the first time that cultural engagement could be a protective psychosocial activity. These results have consequent implications for clinicians working with high risk groups.

Funding: DF is supported by the Wellcome Trust, grant [205407/Z/16/Z].

Conflict of interest: Both authors declare no conflict of interest.

Summary of contributions: DF and AS designed the study. DF carried out the analyses. Both authors worked on the interpretation of the results and write-up.

Early Career Researcher status: Daisy Fancourt is an early career researcher. 